\title{
BRAIN TUMOR PATTERN RECOGNITION USING CORRELATION
}

\section{FILTER}

\author{
Rani Thottungal ${ }^{1}$, T. Mahaprakash ${ }^{2}$ \\ ${ }^{1}$ Professor and Head, EEE Department, Kumaraguru College of Technology, Tamil Nadu, India \\ ${ }^{2} P G$ Scholar, EEE Department, Kumaraguru College of Technology, Tamil Nadu, India
}

\begin{abstract}
This paper presents pattern identification in images which plays an important role in many real time applications worldwide. Different types of filters for pattern recognition have been proposed. But there seems to be growing interest in correlation based pattern recognitions. The reason for this is to design effective recognition system that needed to work on complex image processing applications. There is also a need to process large amount of images in real-time. The brain image processing applications are critical and are to be processed faster. In our approach, for this correlation filters are used which posses good mathematical fundamentals. The correlation filters can be effectively implemented either digitally or optodigitally. The pattern recognition is considered as the basic step for vision systems and efficiency is achieved using correlation filters.
\end{abstract}

Keywords: Pattern recognition, image acquisition, preprocessing, correlation filters.

\section{INTRODUCTION}

The image processing field has varied disciplines and in various real time applications images are the sources which can be easily interpreted by human but not that easy for machines. Many researchers of their interest working to build a strong relationship between images and machines. The gap between human and computer vision are said to be closer as the research facts are very much progressive but still images are challenging tasks for computer vision. The technologies are improved and the complex cognitive capabilities exhibited by human brains are making the machines to respond to the environment even without any prior knowledge on the subject of interest. Research works in machine vision technology has exponentially increased in the past two decades and the reason for this is the availability of cheap cameras and fast processors. The computer vision is the evolving technology which There are many algorithms that are available to work on images. Nowadays images are the most strongest form of sources that are to be processed and typically used to evaluate the environment accurately. So processing an image includes various procedures whereas it depends on the field of application. The research works paves the way for understanding of images by machines. Still the challenging part of image and machine relation is the extraction of the object of interest in the images. The growing interest in automatic pattern recognition[1] proves there is a need for processing images without any human interruption. This kind of analyzing images results enormous solutions for many unsolved real time applications. As a promising factor, techniques using correlation filters reduce the distance between image processing and machine vision systems. Our project proposes a flow that the images are being processed effectively by the vision systems. The images acquired using an image acquisition system are preprocessed and then the correlation filters are applied to identify the image pattern automatically.

The section 2 is about the image acquisition system which is totally based on acquiring images from an object interested environment. Then the section 3 shows the pre-processing step to remove the noises in the images. The section 4 discuss the correlation filter for image pattern recognition. Finally section 5 concludes the proposed approach stating with future works followed by references.

\section{IMAGE ACQUISITION SYSTEM}

The computer vision system works with the images obtained using the acquisition system. The image acquisition system is the preliminary stage of any machine vision systems. The image acquisition system uses devices that are capable of recognizing objects of interest. The devices most often used for image acquisition are cameras and sensors. The cameras are the most effective input devices when dealing with the images. Whereas the sensors are useful in sensing environmental activities. Then moreover sensors are not indulged in processing some form of images but may combine with image capturing devices that uses the infrared sensors for acquiring images. The images that are obtained may undergo various processing stages to achieve different vision capabilities that are required in today's world. However, the images that are inputted to the machine systems, that are not satisfactorily acquired then the systems processing may lead to unexpected outputs and decreases the processing nature of the system. Thus the image acquired plays an important role in 
image analyzing stage of machine vision. If so the images are not in the expected form, the indented processing tasks are not said to be successfully achieved. When it comes to image enhancement, the techniques that allow images to progress[11] are available in more numbers but the machine systems rarely acknowledges the enhanced effects. So the image enhancement techniques are not much suited for the machine vision system processing.

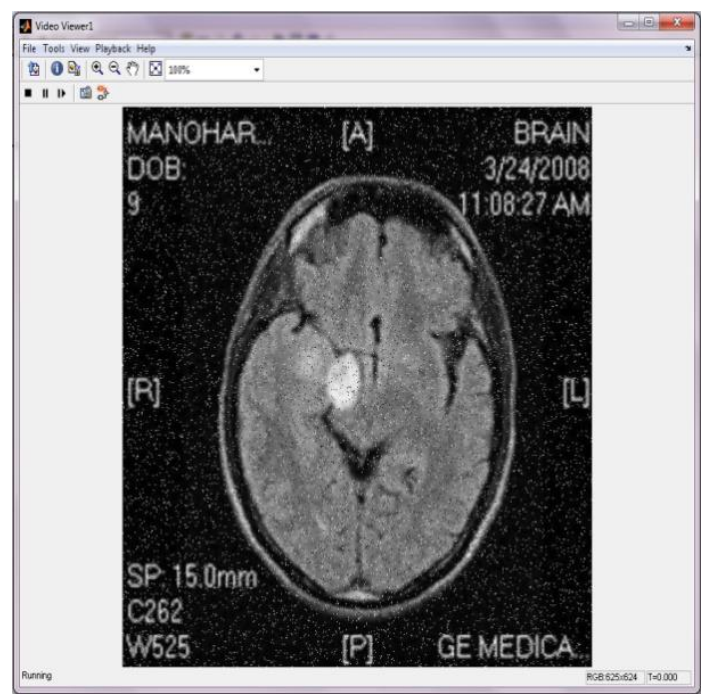

Fig -1 Input Image

As our project, the image acquisition system comprises of a camera which takes a snap and inputs to the image preprocessing stage. As an initial approach we are not used any advanced camera which are capable of motion detections, infrared thermal sensing, etc., But the future works includes the camera setup that is a device which detects any motion with a wide angle of 120 degree and the object of interest in a frame can be obtained using the infrared thermal sensing camera device. The combination above said features are the complete setup for the image acquisition system. This setup provides more accurate information about the object of interest in which the vision system is to be processing.

\section{IMAGE PRE-PROCESSING}

The noise present in the image can reduce the capacity of the region growing filters to grow large regions or may result as faulty edges. When faced with noisy images, it is convenient to pre-process the image by using some kind of filters. These noisy images will lead the vision systems to take wrong decision on the environmental aspects causing damage or lose. This behavior will pay a huge impact on machine vision technology arising a false impression on the system.

The Weighted Median Filter is the most effective filter to remove the noise. The noise presented in the image affects the capacity of region growing filter and it causes problems in the image segmentation process. The noisy image will produce faulty edges on segmentation.

Weighted median filter has the robustness and preserving capability of the classical median filter. Weighted median filter belong to the broad category[2] of non-linear filters called stack filters. This enables the use of the weighted median filter for the noise attenuation capability.

The weighted median filter can be implemented as

$\mathrm{W}(\mathrm{a}, \mathrm{b})=\operatorname{median}\{\mathrm{w} 1 * \mathrm{a} 1 \mathrm{w} 2 * \mathrm{a} 2 \ldots \mathrm{wn} * \mathrm{an}\}$

-- a1.....an are the intensity values inside a window centered at $(\mathrm{a}, \mathrm{b})$ and

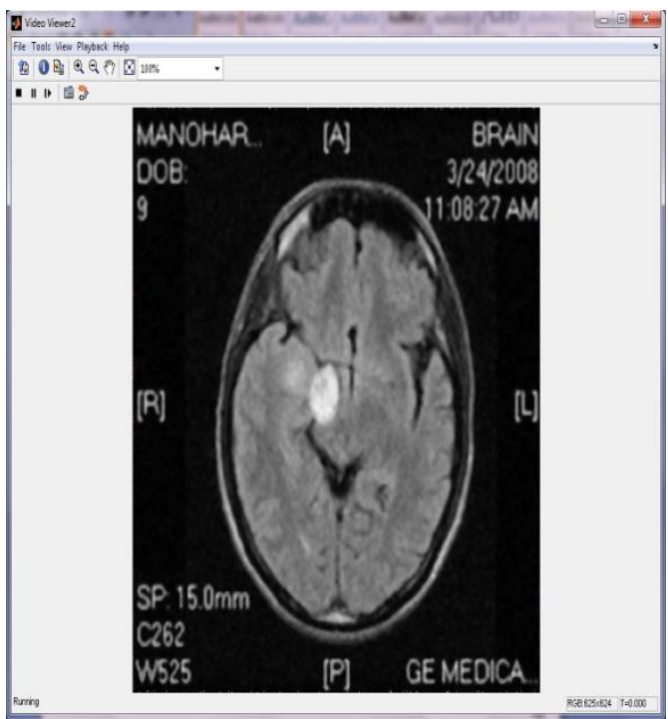

Fig -2: Output Image

-- $\mathrm{w}^{*} \mathrm{n}$ represents the denotes the replication of a, w times.

\section{CORRELATION FILTER}

The pattern recognition includes, different types of tasks that are distinguished essentially into two: identification of a target and evaluation of its exact position. When correlation filters are used for these problems, they are solved in two stages. First, the identification is initiated with searching of correlation peaks in the output, and then peaks coordinates of these filter outputs are considered as position estimates. The quality of both procedures is encountered with the involvement of noise in an prescribed scene. The identification capabilities of correlation filters can be expressed with the use of probability of false errors (detection alarms), SNR ratio, peak-to-output energy ratio, etc,. This also includes discrimination capability of an image[4]. The measures are to be effectively improved with the use of an adaptive approach to design the filter. According to our concept, we are interested to design a filter which shows good performance 
characteristics for a given scene. This can be better explained with a fixed set of patterns and or with a background that is fixed to be rejected than in a filter of average performance parameters over an ensemble of input image scenes. After the identification task has been finished without issues we still faces with small errors of designated positions and estimations because of distortions made of the object by noise. The coordinate estimates lies in the vicinity of their original values. The designated position can be characterized only by means of the variance of measurement errors along coordinates [7].

The most important performance criteria in the pattern recognition approach is that the discrimination capability (DC), or by how means the filter discriminates and detects different types of objects. The correlation filter which has the minimum number of probability of detection errors (false alarms) meant as the optimal filter (OF) [5]. An important feature of the optimal filter is its adaptivity of scene in applications to pattern recognition and/or target identification. The reason because its frequency response considered into account, the power spectrum of error objects in the resulted scene or in the background are to be rejected. The cons of the optimal filter in its implementation is its extreme nature of low light efficiency. A filter with maximum amount of light efficiency is called the phase-only filter (POF)[6]. The limitation of the phase-only filter is its poor discrimination capability for a minimum-low-contrast target embedded into a complexes background scene. An approximation of the optimal filter by means of POF with a quantization was made [8]. The approximate filters with high amount of light efficiency \& the discrimination capability closer to that of the Optimal Filter were discussed. When the object to be identified is in the presence of background noise, the design of the OF was obtained.

The problems of real-time pattern recognition are exploiting adaptive distortion with invariant correlation filters [9]. The distinctive feature of the proposed states the inclusion of an adaptive approach to design the filters. Actually, we will look into two problems: identification of known objects possessing less number of geometric distortions and errors with additive sensor's noise, and design implementation of the filters in an optodigital form. The first and foremost problem is that deciding on presence and absence of a distorted object. The new adaptive filters for reliable recognition of the object in a cluttered background are presented. The adaptive filters also takes into consideration, sensor's noise with a noise realization training. Thus the filters possess a good robustness to the noise. The second issue concerns with the real-time implementation of the adaptive correlation filters. The more critical applications which are some in account, optical or hybrid optodigital techniques are needed and they allow faster processing of images. The pros of optical systems upon computers lies in their ability of optical systems to process data in a parallel way and the classical optical correlated filter allows to perform parallel filtering over an input image containing more patterns. Recent advancement in optical spatial light modulators gives more possibilities for adoption of optodigital systems.

\section{ADAPTIVE CORRELATION FILTERS}

The adaptive correlation filter achieves good recognition of the target. Thus it is necessary to minimize correlation function levels at all wrong peaks except at the initial stage of the correlation plane. For a given object in the image to be recognized, false patterns and backgrounds are to be rejected. It can be carried with the help of an iterative processing algorithm. At each and every iterations, the algorithm minimizes the highest side lobe and then monotonically maximizes the discrimination capability value until a prespecified value will be encountered. The discrimination capability is that filters capable of distinguishing a expected object in an image compared with all other different interested image objects. If a target object is embedded in a background that consist of false objects, then the discrimination capability will be expressed as:

$$
\mathrm{DC}=1-\left\{[\mathrm{Ca}(0,0)]^{2} /[\mathrm{Cr}(0,0)]^{2}\right\}
$$

where $\mathrm{Ca}$ is the peak in the correlation plane around the background area that to be rejected, and $\mathrm{Cr}$ is the peak in the over the area of target location correlation plane. Target position is identified in the close vicinity of the original target position. The background is complementary area to the target position. Negative values of the discrimination capability indicate a tested filter that fails to recognize the output target. We are supposed to design a correlation filter with specifications of identifying a target with a maximum discrimination capability in cluttered and noisy input images. There are conventional correlation filters that they yield a poor performance for this case[10]. So using adaptive correlation filters, a given value of the discrimination capability is achievable. The algorithm to design the filter requires background image knowledge. Thus, we are looking for the target with unknown position in the given input image background. The background can be considered as a realization of a stochastic process, or it characterized to be a picture. The background area can also consist of unknown positioned false objects. The foremost step is to achieve correlation between the background and a basic SDF filter, which is trained with the target initially. The two class recognition is utilized in designing the SDF filter; that is, the true class contains target and the false class contains only false class objects. The proposed iterative flow is repeated until a specified value of the discrimination capability is achieved. If different objects are rejected to be known then they are directly included into the false class. They are used to design the adaptive SDF (ASDF) filter. 
The proposed algorithm consists of the designing the ASDF filter as a conventional SDF filter trained only with the target. Then carrying the correlation between the background and the ASDF filter and determine the discrimination capability. The value of the discrimination capability[3] is greater than or equal to the determined value, then the filter design is finished, if not move to the next stage. The origin of the object in the plane of correlation will be the highest location. The object is included into the false class of objects then design a new filter using two class recognition problem.

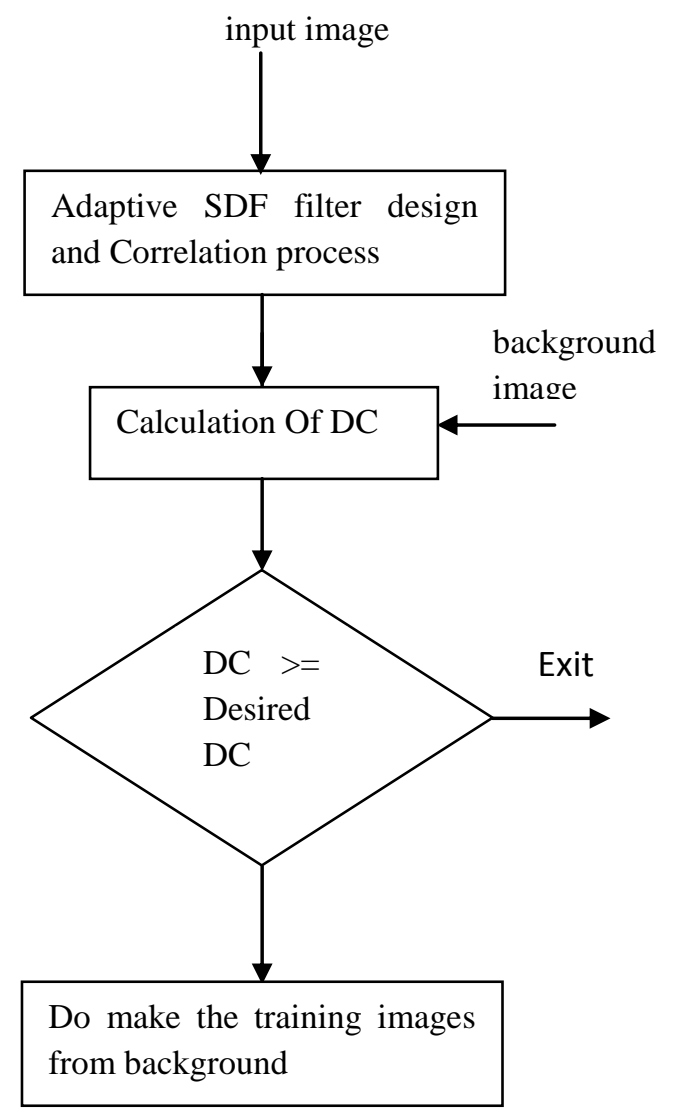

The true class consist of only the target and the false class contains of the false objects. At every iteration, the approach chooses among all other side lobes and such a peak to be minimized in the next step to ensure that the increasing behavior of the discrimination capability versus the iteration index during designing the filter. As a result of the approach, the adaptive composite filter is designed and synthesized. The performance of the filter in recognition process is similar to that of the synthesis process.

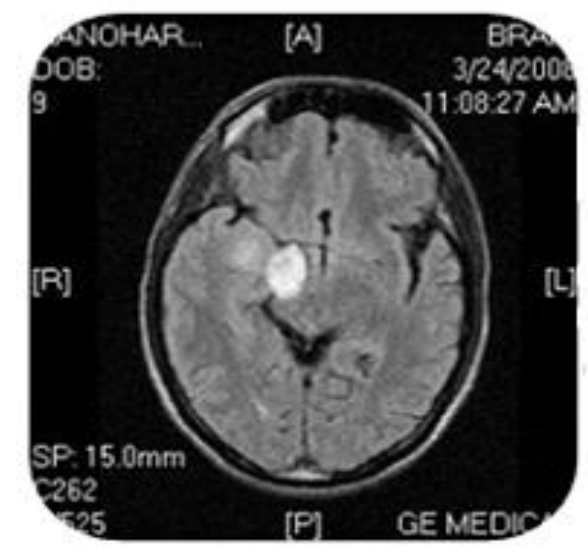

Fig -3: Input Image

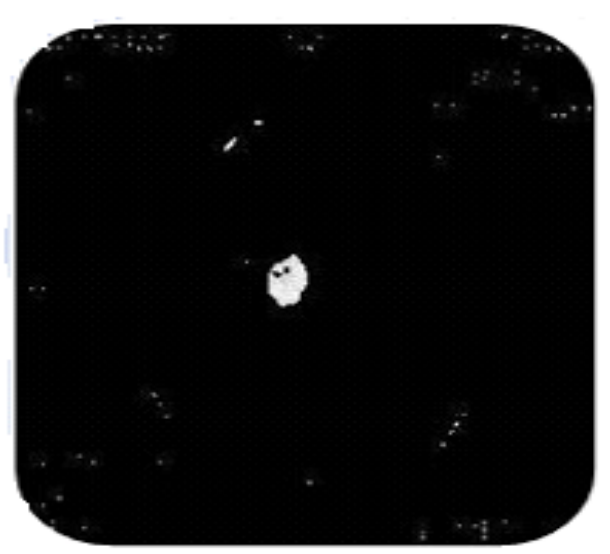

Fig -4: Target Image

From the output figure it is clear that the image acquired and pre-processing steps are done and the correlation filter approach is performed. The performance of the filter shows that the proposed method suits to provide efficient pattern recognition method.

\section{CONCLUSIONS}

The adaptive pattern recognition approach is still in rapid evolution. In our proposed approach, the digital systems designed which is based on adaptive correlation filter to improve objects pattern recognition in a noisy and cluttered backgrounds. The proposed filter design with a training iterations helps control the whole correlation plane. These digital systems are based on iterative training. As a future work the digital systems are replaced with hybrid systems. The hybrid systems are additionally include characteristics of used optoelectronics devices. The digital systems are not able to provide real-time pattern recognition. The results gives a good performance picture of the proposed filters for pattern recognition. The filters are capable of possessing high sceneadaptivity and good robustness to small geometric image distortions and input noise. 


\section{REFERENCES}

[1]. Vitaly Kober, Victor H. Diaz-Ramirez1, J. Angel Gonzalez-Fraga and Josue Alvarez-Borrego. Real-Time Pattern Recognition with Adaptive Correlation Filters. Vision Systems - Chapter. 27 (515-536).

[2]. Arsenault, H. \& Hsu, Y. (1983). Rotation invariant discrimination between almost similar objects, Vol. 22, No. 1, (January 1983) (130-132).

[3]. Billet, O. \& Singher, L. (2002). Adaptive multiple filtering, Vol. 41, No. 1, (January 2002) (55-68).

[4]. Vijaya Kumar, B.V.K. \& Hassebrook, L. (1990). Performance measures for correlation filters, Vol. 29, No. 20, (July 1990) (2997-3006).

[5]. Yaroslavsky, L.P. (1993). The theory of optimal methods for localization of objects in pictures. (145-201), Elsevier.

[6]. Kober V, Mozerov M \& Ovseevich I.A. (2006). Adaptive correlation filters for pattern recognition in Pattern Recognition and Image Analysis, Vol. 16, No. 3, (2006).

[7]. Kober, V. \& Campos, J. (1996). Accuracy of location measurement of a noisy target in a non overlapping background, Vol. 13, No. 8, (August 1996).

[8]. Moreno, I; Campos, J; Yzuel, M.J. \& Kober, V. (1998). Implementation of bipolar real-valued input scenes in a realtime optical correlator: application to color pattern recognition, Vol. 37, No. 1, (January 1998).

[9]. González-Fraga, J. A.; Kober, V. \& Alvarez-Borrego, J. (2006). Adaptive synthetic function filters for pattern recognition, Vol. 45, No. 5, (May 2006).

[10]. Javidi, B. \& Wang, J. (1994). Design of filters to detect a noisy target in non overlapping background noise Vol. 11, No. 10, (October 1994).

[11]. Alam, M. S. \& Kairm, M. A. (1993). Fringe adjusted joint transform correlator, Vol. 32, No. 23, (August 1993).

\section{BIOGRAPHIES}

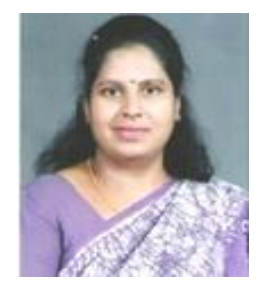

Rani Thottungal, obtained her B.E and M.E degrees from Andhra University and Doctorate from Bharathiar University. She is currently working as Professor and Head in EEE department, Kumaraguru College of Technology, Coimbatore. Her research interest includes Power System, Power Inverter and Power Quality Issues.

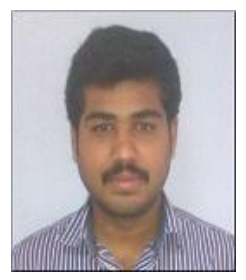

T.Mahaprakash completed his B.E (ECE) in Info Institute of Technology 2012, Coimbatore, Tamil Nadu, India and pursuing final year M.E. in Embedded System in Kumaraguru College of Technology, Coimbatore, Tamil Nadu, India 